\title{
Determinants of housing affordability in the region
}

\author{
Olga Kleshcheva ${ }^{1 *[0000-0001-9198-4014]}$ \\ ${ }^{1}$ Kazan State University of Architecture and Engineering, 420043 Kazan, Russia
}

\begin{abstract}
The issue of providing the population with affordable housing is one of the most pressing social concerns. Each region necessitates a distinct approach. The purpose of this article is to identify the factors that influence a housing affordability level in the region, analyze the impact mechanisms inherent in them, and assess the quantitative impact of these factors on the housing affordability index. The following factors have a direct impact on housing affordability: the rate of inflation, the population size, the cost of housing, the loan interest rate, the rate of housing construction, the investment scale, the income level of the population, and the economic system's overall development level. The quantitative assessment of the cumulative impact of these factors on the level of housing affordability is based on building a regression model describing how the housing affordability index in the region depends on multiple factors and assessing its reliability. The simulation model confirmed that the supply, demand, and housing market conditions have the greatest impact on housing affordability in the region.

Keywords. Affordable housing, housing affordability index, housing policy, simulation, regression model, housing demand, housing market supply.
\end{abstract}

\section{Introduction}

To this day, one of the most pressing social issues is the provision of housing for the population. It appears that identifying the major parameters that determine housing affordability is critical to solving these issues. The basic macroeconomic and political factors influencing the housing market in general are addressed in [1-3]. Torab [4], for example, conducted a detailed analysis of all the factors determining housing affordability in emerging economies. The factors he singled out are: demographics shifts, supply and demand, public policy, inequality and housing, and interest rates. A lot of studies attest to the relationship between housing affordability and changing macroeconomic parameters [5].

Various studies prove that economic cycles determine housing affordability at the state and regional levels, and that it fluctuates due to changes in macroeconomic indicators such as gross national product, income growth, inflation rates, unemployment rates, etc. For example, the authors of [6-9] investigated housing affordability in several regions of the United States and found that unemployment, the federal funds rate, corporate default risk, economic expansion, and unanticipated inflation in the construction market are the major determinants of the housing market. Adams and Fuss looked at statistical data from several

*Corresponding author: olga_albertovna@bk.ru 
countries over a thirty-year period in their research. They discovered that a $1 \%$ change in economic activity levels results in a similar fluctuation in housing demand and a $0.6 \%$ rise in housing prices [8]. Stevenson proved that macroeconomic factors can also account for price fluctuations in the Irish housing market [10].

Changes in income and the value of the interest rate, according to McQuinn and O'Reilly, have the greatest impact on the housing market [1]. Many researchers agree with them, pointing out that the housing market is influenced by changes in loan and mortgage interest rates [11-13]. The relationship between monetary policy tightening and housing prices in the UK, Sweden, and Norway, for example, has been identified [14]. Karpestam and Johansson [15] investigated how prices in the Danish housing market correlate with the mortgage interest rate. Studies of mortgage plan peculiarities in Tatarstan (a Russian region) attest to how this factor influences housing affordability [16].

The impact of irrational factors on pricing processes in the residential real estate market, according to some authors [17], should be recognized. If real estate prices are rising at a fast pace, profiteering may be possible when an apartment is purchased and then sold at a much higher price after a short time. In this case, the price of residential real estate grows even faster and price bubbles in the housing market begin to occur. Gazzani [18] believes that bubbles in the housing market account for a major portion of the housing price fluctuations.

Overseas studies of the main determinants of the housing market have been completed for Lithuania [19]. The authors used the Delphi method to identify the main determinants of housing market fluctuations. Some authors suggest assessing to what extent a particular parameter affects the housing affordability through a univariate regression [20]. Liu and $\mathrm{Wu}$ [21] suggest using a model combining Holt's modified exponential smoothing and whale optimization algorithm to forecast the housing market environment. Alqaralleh and Canepa [22] believe that dynamic asymmetries in the housing market cycle can well be modelled using a logistic smooth transition model. Also, the calculations of prices in the housing market are made using [23] a particular parametrization of the transition function used in the transition equation of a smooth transition autoregressive model which improves the fit in the non-central probability region. Faizullin [24] investigated the factors that simulate the housing affordability market by using the principal component analysis.

The studies dealing with determining the basic determinants that account for fluctuations in housing affordability in Tatarstan cannot now be regarded as complete and exhaustive, so this article aims to analyze how the basic parameters affect housing affordability in the region.

\section{Methods}

An important step in developing measures and identifying ways to improve housing affordability is to study how various factors influence it. These factors are shown in Fig. 1.

\begin{tabular}{|c|c|c|}
\hline \multicolumn{3}{|c|}{ Housing affordability } \\
\hline Income of population & Inflation rate & New housing \\
\hline Unemployment & National currency & \\
\hline Develonment leyel & & Population size \\
\hline $\begin{array}{c}\text { of the economic } \\
\text { system }\end{array}$ & $\begin{array}{l}\text { Investment in } \\
\text { residential }\end{array}$ & Cost of housing \\
\hline & construction & Loan cost \\
\hline
\end{tabular}

Fig. 1. Determinants of housing affordability. 
Table 1. Statistical data to analyze how macroeconomic factors affect housing affordability.

\begin{tabular}{|c|c|c|c|c|c|c|c|c|c|c|c|c|c|c|c|c|c|c|c|c|c|c|c|}
\hline & & \multicolumn{22}{|c|}{ Year } \\
\hline Indicator & 离 & $\stackrel{2}{2}$ & ڤે & ¿̊̀ & $\overrightarrow{\stackrel{\Xi}{े}}$ & ڤิ & 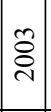 & 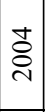 & 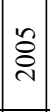 & 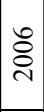 & 客 & 蒿 & ڤેे & 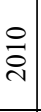 & $\overline{\vec{i}}$ & 글 & $\stackrel{m}{\grave{n}}$ & $\stackrel{ \pm}{\stackrel{\Delta}{\sim}}$ & $\mid \begin{array}{l}n \\
\stackrel{2}{2} \\
\end{array}$ & $\stackrel{\circ}{\circ}$ & 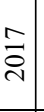 & $\stackrel{\infty}{\stackrel{\sim}{\sim}}$ & हे \\
\hline $\begin{array}{l}\text { Housing affordability } \\
\text { index in the Republic } \\
\text { of Tatarstan, \% [25] }\end{array}$ & $\mathrm{y}$ & $\infty$ & 0 & i & $\tilde{m}$ & $F$ & in & 5 & व & $\begin{array}{l}\infty \\
\infty\end{array}$ & $\infty$ & 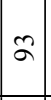 & aे & $\simeq$ & $\stackrel{m}{=}$ & $\stackrel{2}{=}$ & $\stackrel{\partial}{\beth}$ & 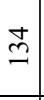 & $\tilde{2}$ & $\hat{n}$ & $\cong$ & t & 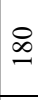 \\
\hline $\begin{array}{l}\text { Housing } \\
\text { commissioning in the } \\
\text { Republic of Tatarstan, } \\
\text { thousand sq. m. of total } \\
\text { floor area [26] }\end{array}$ & $\mathrm{x}_{1}$ & 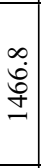 & 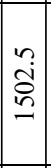 & $\begin{array}{l}0 \\
\dot{\delta} \\
\vdots \\
\end{array}$ & 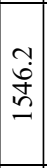 & 竎 & 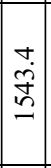 & 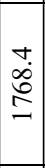 & 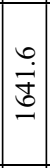 & 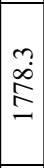 & $\begin{array}{c}r \\
\dot{0} \\
\dot{i}\end{array}$ & 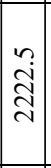 & 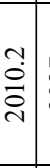 & ธิे & $\begin{array}{l}\overrightarrow{1} \\
0 \\
\text { ते }\end{array}$ & 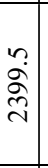 & 苍 & 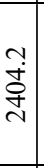 & $\begin{array}{l}0 \\
\dot{d} \\
\stackrel{+}{d} \\
\text { d }\end{array}$ & 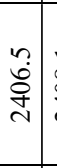 & $\begin{array}{l}\vec{\infty} \\
0 \\
+ \\
\sim \\
\sim\end{array}$ & 高. & 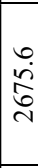 \\
\hline $\begin{array}{l}\text { Size of permanent } \\
\text { population of the } \\
\text { Republic of Tatarstan, } \\
\text { thousand people [26] }\end{array}$ & $\mathrm{x}_{2}$ & $\begin{array}{c} \pm \\
\infty \\
\infty\end{array}$ & $\left|\begin{array}{c}d \\
i \\
\infty \\
\infty \\
m\end{array}\right|$ & $\begin{array}{l}\infty \\
\dot{\infty} \\
\infty \\
\infty \\
m\end{array}$ & $\begin{array}{c}\frac{a}{\infty} \\
\infty \\
\infty \\
m\end{array}$ & 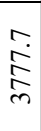 & $\mid \begin{array}{c}\stackrel{a}{i} \\
\stackrel{i}{n} \\
m\end{array}$ & $\begin{array}{l}n \\
0 \\
0 \\
0 \\
n\end{array}$ & $\mid \begin{array}{c}a \\
\vdots \\
0 \\
m\end{array}$ & $\begin{array}{l}\infty \\
\infty \\
\infty \\
n \\
n\end{array}$ & $\mid \begin{array}{c}\infty \\
i \\
i \\
m \\
m\end{array}$ & $\mid \begin{array}{l}0 \\
0 \\
0 \\
0 \\
m\end{array}$ & $\begin{array}{l}n \\
\infty \\
\infty \\
\infty \\
\infty\end{array}$ & $\begin{array}{l}n \\
0 \\
0 \\
0 \\
m \\
m\end{array}$ & $\begin{array}{l}n \\
\infty \\
\infty \\
m\end{array}$ & $\begin{array}{l}c \\
\tilde{b} \\
\infty \\
\infty \\
ల\end{array}$ & $\underset{\substack{\tilde{N} \\
\infty \\
\infty}}{ }$ & $\begin{array}{l}0 \\
\dot{0} \\
\dot{0} \\
\infty \\
m\end{array}$ & 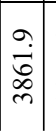 & 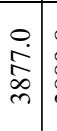 & $\begin{array}{l}\infty \\
0 \\
\infty \\
\infty \\
m\end{array}$ & 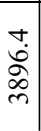 & $\begin{array}{l}\hat{\alpha} \\
\hat{\delta} \\
\text { dे }\end{array}$ \\
\hline $\begin{array}{l}\text { Average prices in the } \\
\text { existing housing market } \\
\text { of the Republic of } \\
\text { Tatarstan, rubles per } 1 \\
\text { sq.m [26] }\end{array}$ & $x_{3}$ & 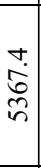 & \begin{tabular}{c}
$\tilde{Z}$ \\
\multirow{\sigma}{*}{}
\end{tabular} & 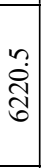 & $\begin{array}{l}m \\
\infty \\
\infty \\
\infty \\
\infty\end{array}$ & $\begin{array}{l}\stackrel{t}{\circ} \\
\stackrel{\sigma}{\sigma}\end{array}$ & 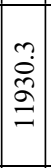 & 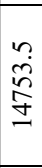 & 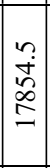 & $\mid \begin{array}{c}0 \\
0 \\
0 \\
0 \\
m\end{array}$ & $\mid \begin{array}{c}\infty \\
\dot{m} \\
m \\
m \\
m\end{array}$ & $\mid \begin{array}{c}\frac{a}{2} \\
f \\
f\end{array}$ & 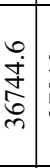 & 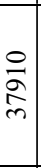 & $\begin{array}{l}0 \\
\stackrel{2}{c} \\
\text { ch }\end{array}$ & $\begin{array}{l}\overrightarrow{\widehat{D}} \\
\stackrel{?}{+}\end{array}$ & $\begin{array}{l}\text { సे } \\
\text { مू } \\
\text { n. }\end{array}$ & $\begin{array}{l}\hat{n} \\
\hat{d} \\
n\end{array}$ & $\begin{array}{l}\infty \\
0 \\
0 \\
0 \\
n \\
n\end{array}$ & $\begin{array}{l}\text { 文 } \\
\text { ì } \\
\text { in }\end{array}$ & $\begin{array}{l}\overrightarrow{7} \\
\stackrel{n}{n} \\
n\end{array}$ & लై & 胥 \\
\hline \begin{tabular}{|l|} 
Average prices in the \\
new housing market of \\
the Republic of \\
Tatarstan, rubles per 1 \\
$\mathrm{~m}^{2}[26]$ \\
\end{tabular} & $\mathrm{x}_{4}$ & $\begin{array}{l}\vec{i} \\
\vec{d} \\
\dot{\sigma}\end{array}$ & $\begin{array}{l}\dot{a} \\
\vec{i} \\
\infty \\
n\end{array}$ & $\begin{array}{l}\hat{i} \\
\stackrel{f}{0}\end{array}$ & $\mid \begin{array}{l}n \\
\infty \\
2 \\
2\end{array}$ & $\begin{array}{l}0 \\
\stackrel{0}{S} \\
\frac{a}{\sigma}\end{array}$ & $\mid \begin{array}{l}n \\
0 \\
0 \\
0 \\
0 \\
1\end{array}$ & $\stackrel{n}{\stackrel{n}{\hat{a}}}$ & $\begin{array}{c}a \\
\infty \\
\tilde{n} \\
\underline{n}\end{array}$ & $\begin{array}{l}r \\
\infty \\
\infty \\
\stackrel{2}{v}\end{array}$ & 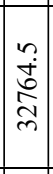 & $\begin{array}{l}8 \\
\stackrel{1}{7} \\
\forall\end{array}$ & 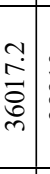 & 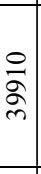 & $\begin{array}{l}\hat{\sim} \\
\stackrel{\sigma}{\gamma}\end{array}$ & 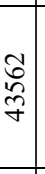 & 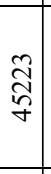 & 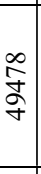 & 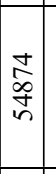 & 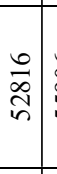 & 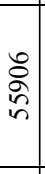 & 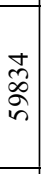 & 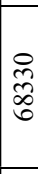 \\
\hline $\begin{array}{l}\text { Gross Regional Product } \\
\text { of the Republic of } \\
\text { Tatarstan, million } \\
\text { rubles [26] }\end{array}$ & $\mathrm{x}_{5}$ & 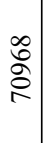 & $\begin{array}{l}8 \\
\stackrel{2}{\Xi} \\
=\end{array}$ & \begin{tabular}{l}
\multirow{2}{n}{} \\
$\overrightarrow{0}$ \\
$\infty$ \\
-
\end{tabular} & 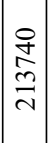 & 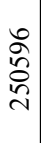 & $\begin{array}{l}0 \\
0 \\
0 \\
0 \\
0 \\
0\end{array}$ & $\begin{array}{l}0 \\
\cdots \\
\bar{\sigma} \\
\text { m }\end{array}$ & $\mid \begin{array}{c}8 \\
8 \\
0 \\
\infty \\
\infty \\
+ \\
+\end{array}$ & $\begin{array}{l}n \\
n \\
n \\
z \\
\delta\end{array}$ & 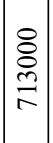 & 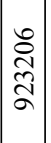 & 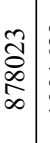 & 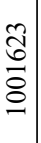 & $\begin{array}{l}\text { y } \\
2 \\
0 \\
0 \\
0\end{array}$ & 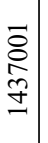 & 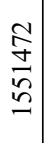 & 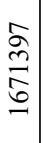 & $\mid \begin{array}{c}0 \\
0 \\
0 \\
i \\
0 \\
0 \\
-1\end{array}$ & 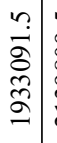 & $\begin{array}{l}n \\
\dot{2} \\
\infty \\
2 \\
\\
\end{array}$ & 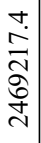 & 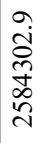 \\
\hline $\begin{array}{l}\text { Size of unemployed } \\
\text { population of the } \\
\text { Republic of Tatarstan, } \\
\text { thousand people [26] }\end{array}$ & $\mathrm{x}_{6}$ & $\begin{array}{l}n \\
\infty \\
\infty \\
=\end{array}$ & 范 & $\mid \begin{array}{l}n \\
\infty \\
n \\
n\end{array}$ & $\vec{b}$ & $\hat{\sigma}$ & 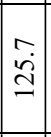 & 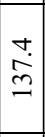 & 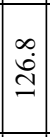 & $\hat{0}$ & $\mid \begin{array}{c}3 \\
0 \\
0 \\
0\end{array}$ & नें & อ으 & 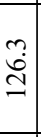 & $\dot{a}$ & $\begin{array}{l}0 \\
\dot{\infty} \\
\dot{\infty}\end{array}$ & $\hat{\dot{\infty}}$ & $\begin{array}{l}\infty \\
\dot{0} \\
\infty \\
\infty\end{array}$ & $\infty$ & 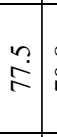 & $\begin{array}{l}\infty \\
\stackrel{0}{r}\end{array}$ & i. & فे \\
\hline $\begin{array}{l}\text { Real cash income of the } \\
\text { population in the } \\
\text { Republic of Tatarstan, } \\
\text { in \% to the last year } \\
{[26]}\end{array}$ & $\mathrm{x}_{7}$ & $\infty$ & $\infty$ & $\Xi$ & $\stackrel{\tilde{I}}{\Xi}$ & $\begin{array}{l}\stackrel{0}{\mathfrak{J}} \\
\stackrel{\Xi}{\Xi}\end{array}$ & $\begin{array}{l}\dot{v} \\
\stackrel{\Xi}{\Xi}\end{array}$ & 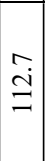 & $\mid \begin{array}{c}m \\
\vec{a} \\
=\end{array}$ & 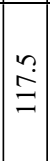 & $\mid \begin{array}{l}n \\
\stackrel{\Xi}{\Xi} \\
=\end{array}$ & $\stackrel{\stackrel{0}{0}}{\varrho}$ & 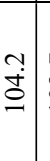 & 。ㅇ & $\stackrel{\infty}{\infty}$ & $\stackrel{\sim}{\stackrel{\Xi}{\Xi}}$ & $\begin{array}{l}n \\
\text { ci } \\
0\end{array}$ & 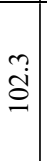 & $\begin{array}{l}0 \\
\dot{a}\end{array}$ & ชู้ & ஜ̊. & 官 & $\stackrel{\overbrace{}}{0}$ \\
\hline $\begin{array}{l}\text { Refinancing rate (key } \\
\text { rate) of the Central } \\
\text { Bank of the Russian } \\
\text { Federation, \% [27] }\end{array}$ & $\mathrm{x}_{8}$ & $\hat{n}$ & in & $m$ & $\stackrel{2}{2}$ & $\tilde{\lambda}$ & $=$ & \pm & $\cong$ & $\simeq$ & 9 & $=$ & $\simeq$ & $\stackrel{2}{\stackrel{2}{r}}$ & $\infty$ & \begin{tabular}{l}
2 \\
\multirow{\infty}{\infty}{} \\
$\infty$
\end{tabular} & $\mid$\begin{tabular}{l}
$n$ \\
\hdashline \\
$n$
\end{tabular} & $\begin{array}{l}n \\
\varrho \\
\varrho\end{array}$ & $\begin{array}{l}\hat{n} \\
\infty \\
\infty\end{array}$ & $\stackrel{\stackrel{\bullet}{0}}{\circ}$ & $\vec{a}$ & $\begin{array}{c}\infty \\
\stackrel{\infty}{?} \\
?\end{array}$ & $\stackrel{\vec{N}}{r}$ \\
\hline $\begin{array}{l}\text { US dollar } \\
\text { (annual a }\end{array}$ & $\mathrm{X}_{9}$ & 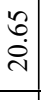 & $\begin{array}{l}\mathfrak{n} \\
\stackrel{v}{\sim}\end{array}$ & $\begin{array}{c}0 \\
0 \\
\infty \\
i \\
i\end{array}$ & $\begin{array}{c} \pm \\
\dot{0} \\
\dot{m}\end{array}$ & 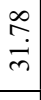 & $\begin{array}{l}n \\
\stackrel{n}{2} \\
\stackrel{2}{2}\end{array}$ & $\begin{array}{l}\cong \\
\stackrel{\sim}{\sim}\end{array}$ & $\left|\begin{array}{l}\infty \\
2 \\
\infty \\
\sim \\
\sim\end{array}\right|$ & 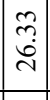 & $\mid \begin{array}{l}n \\
n \\
\dot{\tau}\end{array}$ & 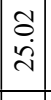 & $\begin{array}{l}n \\
\stackrel{n}{n} \\
\stackrel{n}{n}\end{array}$ & 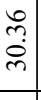 & ले & 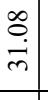 & $\mid \begin{array}{c}\infty \\
\infty \\
\dot{m}\end{array}$ & $\begin{array}{l}\vec{b} \\
\infty \\
\infty\end{array}$ & $\begin{array}{c}\tilde{0} \\
\stackrel{0}{6}\end{array}$ & $\begin{array}{l}\infty \\
\varnothing \\
\varnothing \\
\varnothing\end{array}$ & $\begin{array}{l}m \\
\infty \\
i n\end{array}$ & $\begin{array}{l}\tilde{\hat{~}} \\
\text { }\end{array}$ & $\begin{array}{l}\mathcal{b} \\
\dot{b}\end{array}$ \\
\hline $\begin{array}{l}\text { Consumer price indices } \\
\text { in the Republic of } \\
\text { Tatarstan, \% [26] }\end{array}$ & $\mathrm{x}_{10}$ & $\begin{array}{l}m \\
\stackrel{2}{2} \\
\end{array}$ & $\left|\begin{array}{l}n \\
\infty \\
\infty \\
n\end{array}\right|$ & 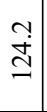 & 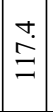 & $\begin{array}{l}\stackrel{\nabla}{\bullet} \\
\stackrel{=}{=}\end{array}$ & 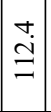 & $\underline{\underline{\mathrm{J}}}$ & $\mid \begin{array}{l}\infty \\
0 \\
\infty \\
0\end{array}$ & 它. & $\mid \begin{array}{l}0 \\
\doteq \\
=\end{array}$ & 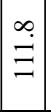 & 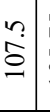 & $\begin{array}{l}n \\
\\
\dot{c} \\
\varrho\end{array}$ & $\begin{array}{l}2 \\
0 \\
0 \\
0\end{array}$ & 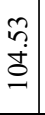 & $\left|\begin{array}{l}n \\
\vdots \\
o \\
0\end{array}\right|$ & $\begin{array}{l}\overrightarrow{\hat{a}} \\
\dot{0}\end{array}$ & $\stackrel{\ddot{0}}{\varrho}$ & હे & 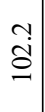 & 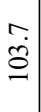 & $\widehat{\hat{\delta}}$ \\
\hline $\begin{array}{l}\text { Investment in fixed } \\
\text { capital by activity type } \\
\text { «Construction» in the } \\
\text { Republic of Tatarstan, } \\
\text { million rubles [26] }\end{array}$ & $\mathrm{x}_{11}$ & ลे & 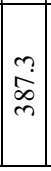 & ¿ें & $\begin{array}{c}n \\
6 \\
-\end{array}$ & 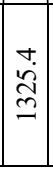 & $\begin{array}{l}-1 \\
\dot{0} \\
2\end{array}$ & $\begin{array}{c}\overrightarrow{\mathrm{i}} \\
\stackrel{\infty}{\infty} \\
-\end{array}$ & 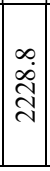 & 总 & 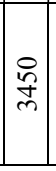 & $\begin{array}{l}0 \\
8 \\
\infty \\
\infty\end{array}$ & in & వి & $\ddot{\check{\alpha}}$ & 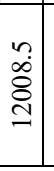 & $\mid \begin{array}{c}0 \\
\infty \\
0 \\
\vdots \\
\vdots\end{array}$ & 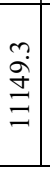 & $\begin{array}{l}2 \\
\tilde{1} \\
0 \\
\delta \\
0\end{array}$ & 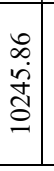 & $\begin{array}{l}n \\
n \\
\frac{2}{2}\end{array}$ & $\begin{array}{l}\stackrel{0}{0} \\
\stackrel{n}{n} \\
i n\end{array}$ & $\begin{array}{l}\stackrel{\partial}{\mathrm{b}} \\
\stackrel{\sigma}{\sigma}\end{array}$ \\
\hline
\end{tabular}

The principal determinant of the housing affordability level in the region is household income. This factor is recognized in the calculations of a housing affordability index. The inflation rate has a great impact on the real income of households: when the inflation rate is 
high, housing becomes less affordable. Since income is earned by a working population, housing affordability suffers when unemployment is high. The level of housing prices also has a direct impact on housing affordability. If prices are high, housing affordability goes down. If there is an increase in the number of houses to be commissioned, prices may fall somewhat, which will make housing more affordable. Growing investment in construction, in turn, causes more housing to be commissioned. Investment amounts will vary according to the growth level of the economy.

The size of the population has a direct effect on housing demand. The availability of various lending and mortgage plans is a determining factor for most people when buying dwellings. The national currency exchange rate dictates the cost of imported building materials and influences the final price on the new housing market.

In studying the issues of improving the housing affordability, a quantitative analysis of the impact of various factors is important. The Housing Affordability Index is an indicator of housing affordability (Table 1). This indicator is the ratio of the income of an average household to the income sufficient to buy a standard apartment through a mortgage loan issued on standard terms [25]. If this value is higher than $100 \%$, this means that the income of the average household is equal to the income sufficient to buy a standard apartment through a mortgage loan. A value below 100\% suggests that an average household is unable to buy housing. Looking at how this indicator has changed in the Republic of Tatarstan over time, we can make a conclusion about housing affordability for an average household starting from 2010 and up to now.

Table 1 shows the dynamics of the determinants of housing affordability as well.

The partial least squares regression method was used to create a model of the dependence of the housing affordability index on different factors. When this method is applied, the matrix of factors and the matrix of resulting indicators first undergo an interdependent decomposition using the principal component analysis. This allows only relevant information to be made more accurate. Then a regression model of the resulting factor's dependence on the principal components is built.

The calculation algorithm for the model by partial least squares regression involves the following calculations:

Finding the principal components means building a model:

$$
\begin{aligned}
& \mathbf{X}=\mathbf{T} \mathbf{P}^{\mathrm{T}}+\mathbf{E}, \\
& \mathbf{Y}=\mathbf{U} \mathbf{Q}^{\mathrm{T}}+\mathbf{F},
\end{aligned}
$$

where $\mathbf{X}$ is the matrix of input data using the factor attributes:

$\mathbf{Y}$ is the matrix of input data using the resultant attributes;

$\mathbf{T}$ is the matrix of scores of the input data using the factor attributes;

$\mathbf{U}$ is the matrix of scores of input data using the resultant attributes;

$\mathbf{P}$ is the matrix of loads of the input data using the factor attributes;

$\mathbf{Q}$ is the matrix of loads of input data using the resultant attributes;

$\mathbf{E}$ is the matrix of residues of the input data using the factor attributes;

$\mathbf{F}$ is the matrix of residues of input data using the resultant attributes.

Input data are normalized and aligned. Then, for each principal component, we calculate the values of scores (coordinates in the principal component space) and loads (transition vectors from the input data space to the principal component space):

1. Using the least square method, we calculate the values of weighted loads $\mathbf{w}_{\mathrm{a}}$ :

$$
\mathbf{X}_{\mathrm{a}-1}=\mathbf{y}_{\mathrm{a}-1} \mathbf{w}_{\mathrm{a}}^{\mathrm{T}}+\mathbf{E}
$$

2. Scores $\hat{\mathbf{t}}_{\mathrm{a}}$ are found:

$$
\begin{gathered}
\mathbf{X}_{\mathrm{a}-1}=\mathbf{t}_{\mathrm{a}} \hat{\mathbf{w}}_{\mathrm{a}}^{\mathrm{T}}+\mathbf{E} \\
\hat{\mathbf{t}}_{\mathrm{a}}=\mathbf{X}_{\mathrm{a}-1} \hat{\mathbf{w}}_{\mathrm{a}}
\end{gathered}
$$


3.Loads $\mathbf{p}_{\mathrm{a}}$ are determined:

$$
\begin{gathered}
\mathbf{X}_{\mathrm{a}-1}=\hat{\mathbf{t}}_{\mathrm{a}} \mathbf{p}_{\mathrm{a}}^{\mathrm{T}}+\mathbf{E} \\
\hat{\mathbf{p}}_{\mathrm{a}}=\mathbf{X}_{\mathrm{a}-1}^{\mathrm{T}} \hat{\mathbf{t}}_{\mathbf{a}} / \hat{\mathbf{t}}_{\mathrm{a}}^{\mathrm{T}} \hat{\mathbf{t}}_{\mathrm{a}}
\end{gathered}
$$

4. Loads $\mathbf{q}_{\mathrm{a}}$ are calculated:

$$
\begin{array}{r}
\mathbf{y}_{\mathrm{a}-1}=\hat{\mathbf{t}}_{\mathrm{a}} \mathbf{q}_{\mathrm{a}}+\mathbf{f} \\
\hat{\mathbf{q}}_{\mathrm{a}}=\mathbf{y}_{\mathrm{a}-1}^{\mathrm{T}} \hat{\mathbf{t}}_{\mathrm{a}} / \hat{\mathbf{t}}_{\mathrm{a}}^{\mathrm{T}} \hat{\mathbf{t}}_{\mathrm{a}}
\end{array}
$$

5. $\mathbf{X}$ and $\mathbf{Y}$ residues are found:

$$
\begin{aligned}
\hat{\mathbf{E}} & =\mathbf{X}_{\mathrm{a}-1}-\hat{\mathbf{t}}_{\mathrm{a}} \hat{\mathbf{p}}_{\mathrm{a}}^{\mathrm{T}} \\
\hat{\mathbf{f}} & =\mathbf{y}_{\mathrm{a}-1}-\hat{\mathbf{t}}_{\mathrm{a}} \hat{\mathbf{q}}_{\mathrm{a}}
\end{aligned}
$$

6. $\hat{b}_{0}$ and $\hat{\mathbf{b}}$ values are determined for the regression equation:

$$
\begin{gathered}
\hat{\mathbf{y}}=\mathbf{1} \hat{b}_{0}+\mathbf{X} \hat{\mathbf{b}} \\
\hat{\mathbf{b}}=\hat{\mathbf{W}}\left(\hat{\mathbf{P}}^{\mathrm{T}} \hat{\mathbf{W}}\right)^{-1} \hat{\mathbf{q}} \\
\hat{b}_{0}=\bar{y}-\overline{\mathbf{x}}^{\mathrm{T}} \hat{\mathbf{b}}
\end{gathered}
$$

\section{Results and discussions}

The calculation of the values of the residual variance indicator determined that the first three principal components are required to build a regression model. They describe $96 \%$ of the variation in the Housing Affordability Index in Tatarstan.

The use of the partial least square's regression involves the calculation and analysis of loads and scores based on the model built. Loads give the information on the direction of the principal components. The greater the absolute value of the loads on the factor, the stronger this factor affects the direction of the principal component. Scores are the projections that the original economic periods make onto the principal component. If we analyze the values of loads and scores for the first principal component (Fig. 2-3), we can infer the economic interpretation for it.

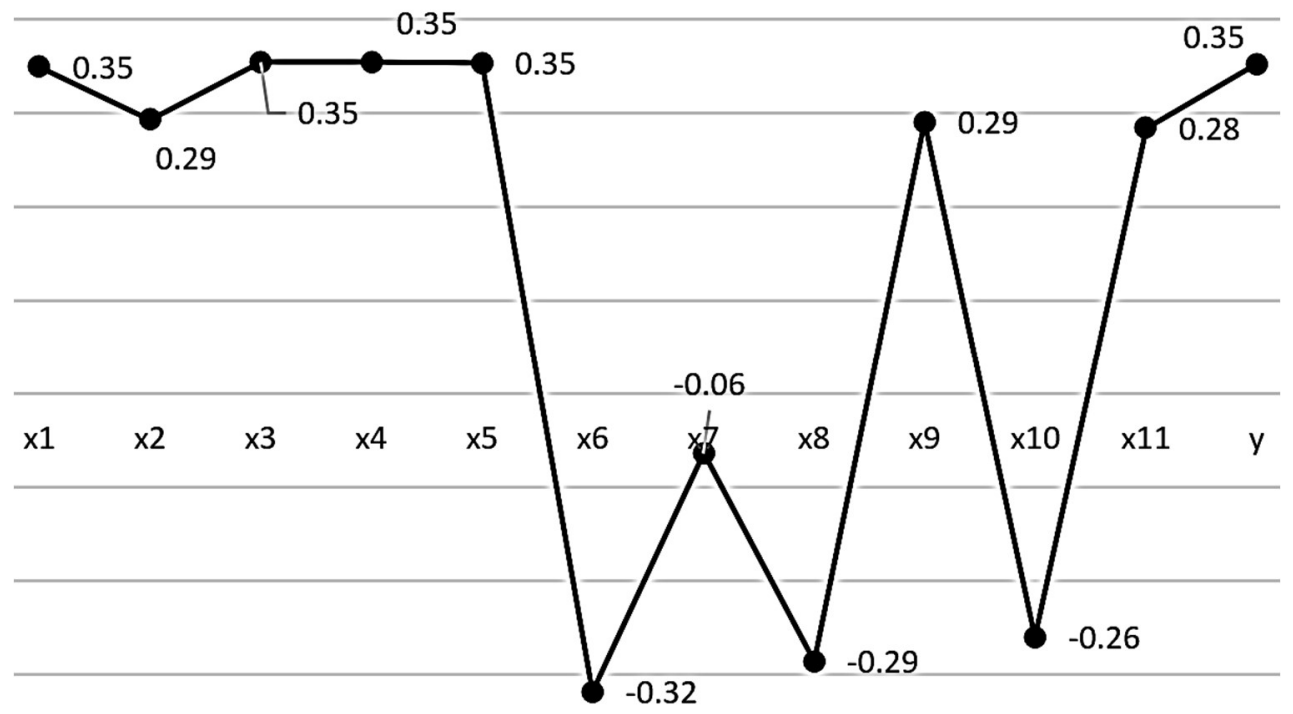

Fig. 2. Load values for the first principal component (See Table 1 for symbols). 
The factors that have a determining influence on the direction of the first principal component (the loads are given in parentheses) are housing commissioning (0.35), average prices on the existing housing market (0.35), average prices in the new housing market $(0.35)$, gross regional product $(0.35)$, and the housing affordability index $(0.35)$. This principal component is interpreted as a supply factor in the housing market. The analysis of the chart of scores confirms the interpretation of the first principal component.

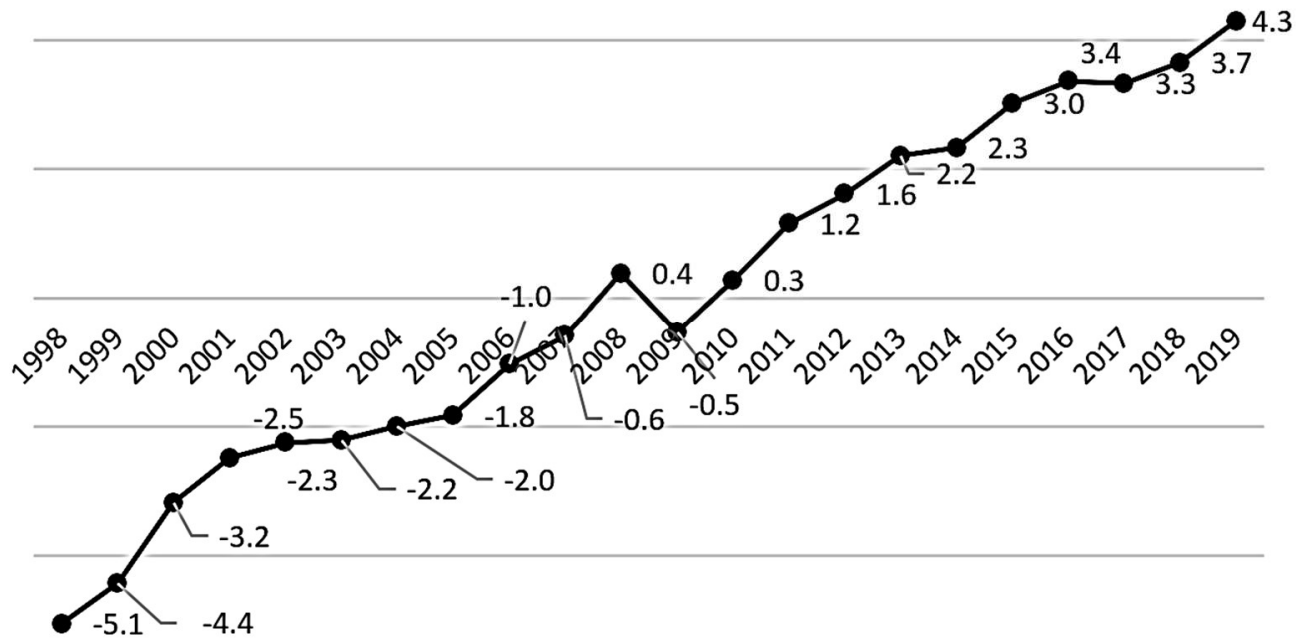

Fig. 3. Score values for the first principal component.

The factors that have the major impact on the direction of the second principal component are the resident population size (-0.4), real money income of the population (0.9), a key rate $(-0.55)$, and consumer price indices (-0.6) (Fig. 4).

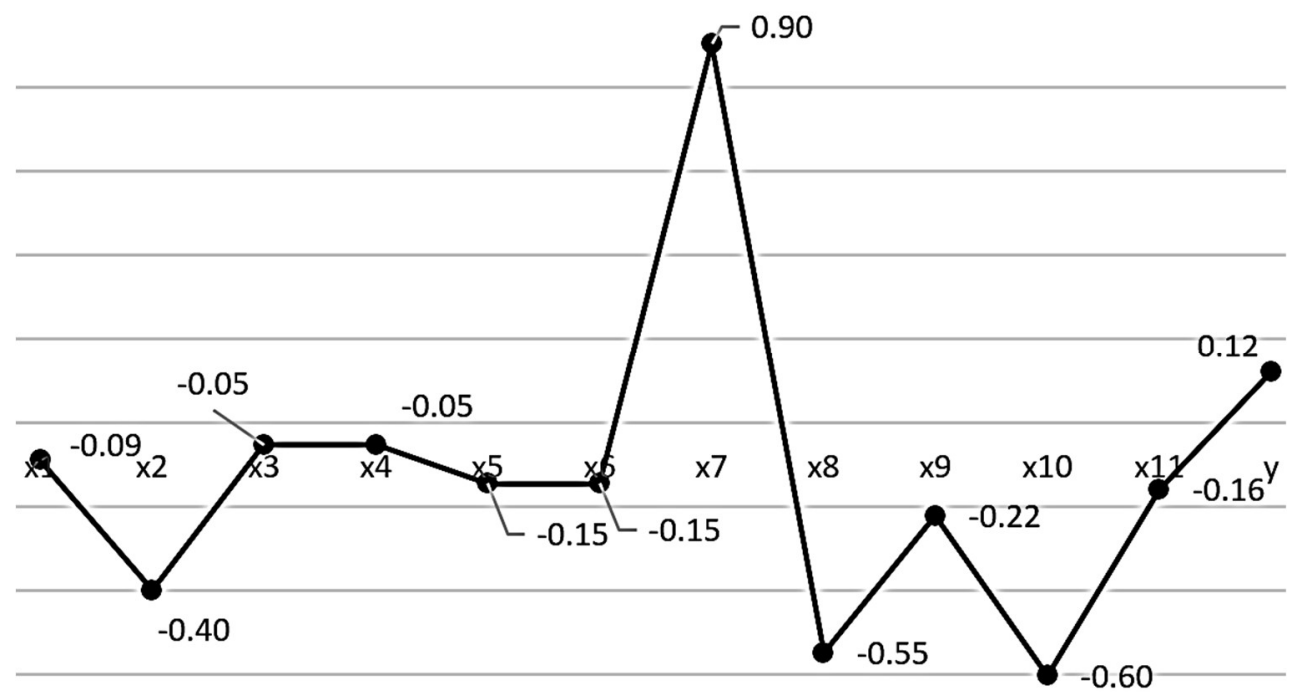

Fig. 4. Load values for the second principal component.

The second principal component is interpreted as a housing demand factor. This interpretation is confirmed by the analysis of the chart of scores (Fig. 5). 


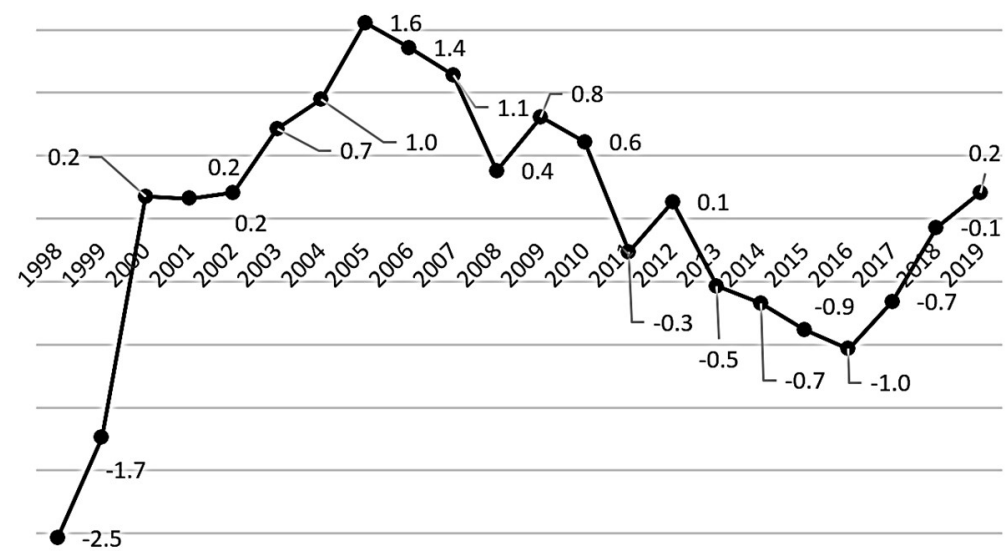

Fig. 5. Score values for the second principal component.

The third principal component is shaped by the following factors: the number of unemployed (0.4), real money income of the population $(-0.41)$, the dollar exchange rate (0.4), and investment in fixed capital of construction companies (-0.62) (Fig. 6).

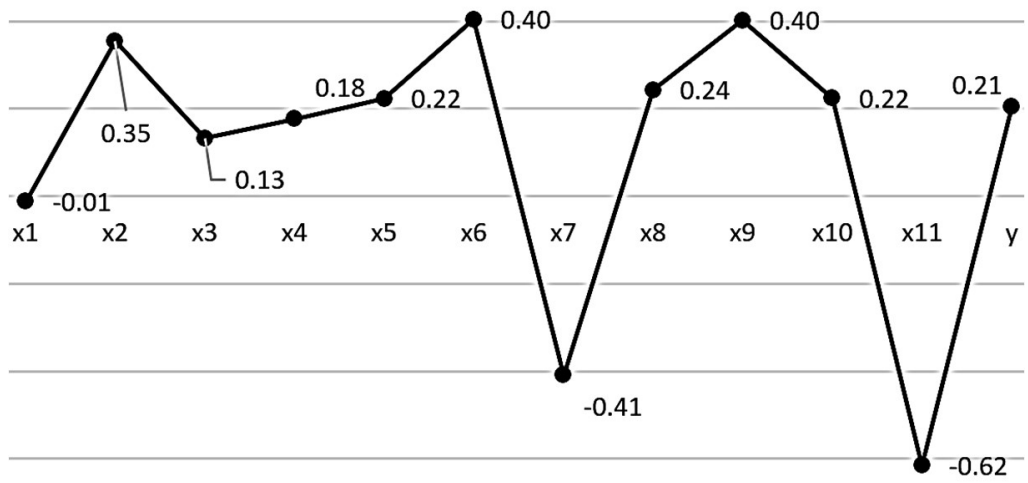

Fig. 6. Load values for the third principal component.

The third principal component is interpreted as the housing market environment. The chart of scores (Fig. 7) shows how the market environment fluctuates.

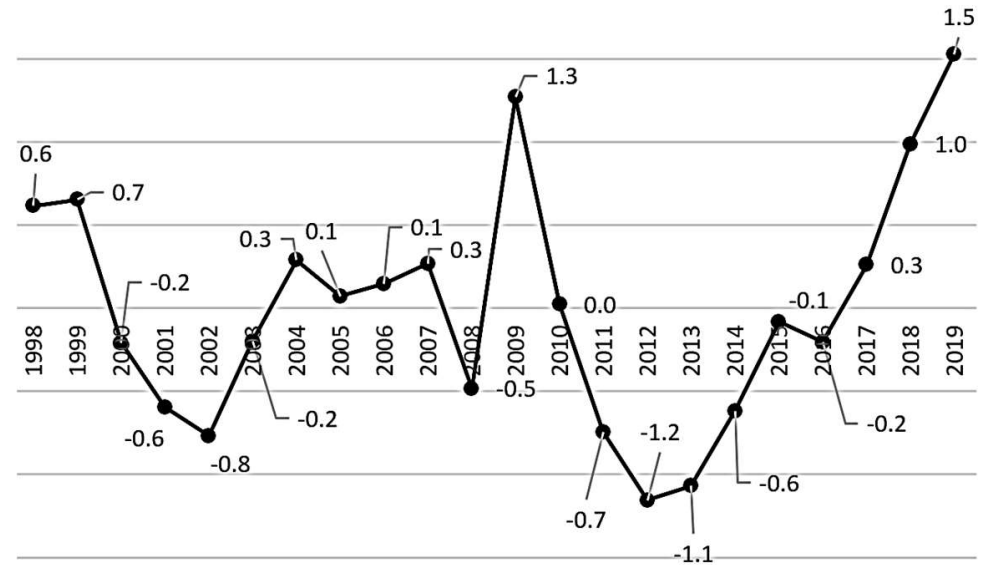

Fig. 7. Score values for the third principal component. 
The analysis resulted in the following regression model:

$y=0.0168 \cdot x_{1}+0.0827 \cdot x_{2}+0.0004 \cdot x_{3}+0.0005 \cdot x_{4}+0.00001 \cdot x_{5}+0.112 \cdot x_{6}+0.199 \cdot x_{7-}$

$-0.721 \cdot x_{8}+0.29 \cdot x_{9}-0.394 \cdot x_{10}-0.0016 \cdot x_{11}-279.837$.

The determination coefficient $\left(\mathrm{R}^{2}\right)$ was calculated to assess the adequacy of the model built. It was 0.94: the model built accounts for $94 \%$ of the fluctuations of the resultant factor, and is therefore reasonable for the analysis. Figure 8 shows the dynamics of input values of the housing affordability index in Tatarstan and the dynamics of the values that were calculated with the regression model. The average approximation error is $14 \%$; this indicates that the regression model is reasonable for the analysis.

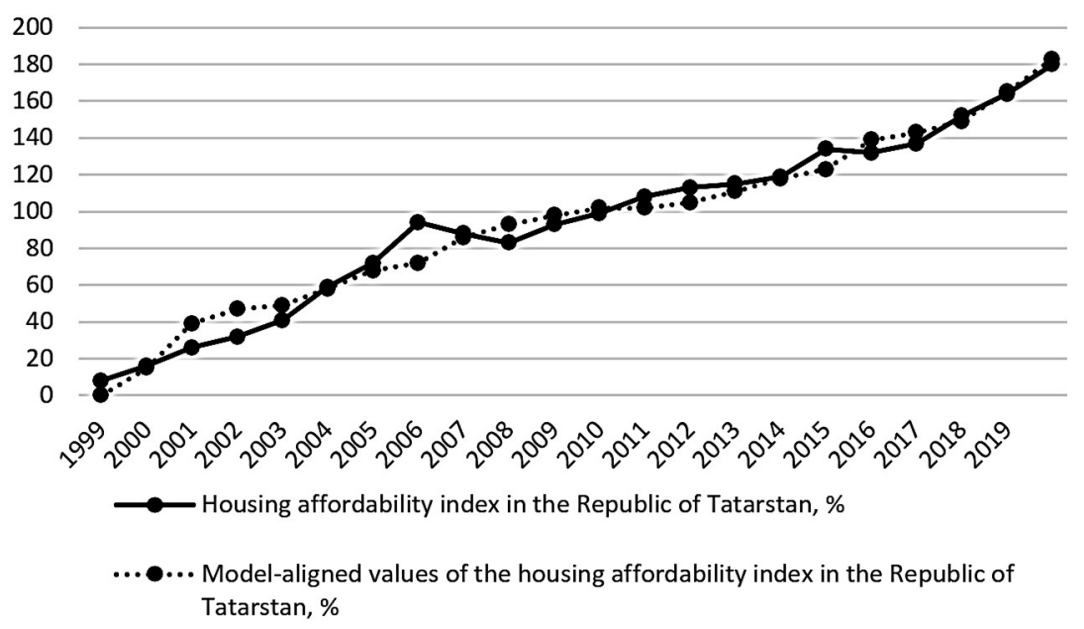

Fig. 8. Input values vs. model-aligned values of the housing affordability index in Tatarstan.

In general, the differences between the housing affordability index calculated using the housing affordability model and the real value are minor. This suggests that the model built represents the real correlation between the housing affordability index in the region and the factors being studied. The model can be used to predict overall housing affordability trends in Tatarstan. The key activities of the region's housing policy can be shaped using this model.

Various studies of the issue of identifying the determinants of affordable housing have been undertaken in different economic systems [19-22]. This study suggests the model that has been calculated for a specific economic system and adjusted for all of its local features. As a result, its use in setting up housing policy in the region can be trusted. The method for calculating model parameters allowed for sufficient accuracy in the simulation.

\section{Conclusion}

It can be inferred from this analysis that housing affordability is influenced directly by the following factors: inflation rates, population size, cost of housing, loan interest rates, housing construction rates, investment rates, population incomes, unemployment rates, national currency exchange rates and the development level of the economic system as a whole.

The regression analysis was applied to assess the effect of these factors in a quantitative way. Using it as a basis, we built a model of the dependence of the housing affordability index on different factors. The simulation data indicate that supply, demand, and the housing market climate have a deciding effect on housing affordability in the region.

In this regard, to make housing more affordable, these steps are required: keep developing the domestic production of construction materials; implement projects for the construction of low-cost housing; implement programs to support low-rise housing projects; implement 
employment promotion incentives; adjust the lending rate through the programs that make housing more affordable to various population groups.

\section{References}

1. K. McQuinn, G. O'Reilly. Economic Modelling 25, 377-390 (2008). DOI: 10.1016/j.econmod.2007.06.010.

2. A. Kaklauskas, A. Daniunas, A. Binkyte, R. Kliukas, P. Kazokaitis, G. Kaklauskas, A. Juozapaitis, A. Banaitis, L. Budryte. Land Use Policy 48, 25-37 (2015). DOI: 10.1016/j.landusepol.2015.05.007.

3. T. Nuuter, I. Lill, L. Tupenaite. Land Use Policy 42, 642-651 (2015). DOI: 10.1016/j.landusepol.2014.09.022.

4. E.S. Torab. Alexandria Engineering Journal 57 (4), 4081-4090 (2018). DOI: 10.1016/j.aej.2018.10.010.

5. N. Yunus. Journal of International Financial Markets, Institutions \& Money 36, 100-112 (2015). DOI: 10.1016/j.intfin.2014.12.008.

6. L. Agnello, L. Schuknecht. Journal of Housing Economics 20 (3), 171-190 (2011). DOI: 10.1016/j.jhe.2011.04.001.

7. E.R. de Wit, P. Englund, M.K. Francke. Regional Science and Urban Economics 43 (2), 220-241 (2013). DOI: 10.1016/j.regsciurbeco.2012.07.002.

8. Z. Adams, R. Fuss. Journal of Housing Economics, 19 (1), 38-50 (2010). DOI: 10.1016/j.jhe.2009.10.005

9. M.L. Fadiga, Y.Y. Wang. Journal of Economics and Finance 33, 13-26 (2009). DOI: 10.1007/s12197-008-9027-5.

10. S. Stevenson. Real Estate Economics 36 (1), 1-29 (2008). DOI: 10.1111/j.15406229.2008.00204.x

11. C.-B. Tse, T. Rodgers, J. Niklewski. Economic Modelling 37, 518-530 (2014). https://doi.org/10.1016/j.econmod.2013.08.013

12. J.J. Su, W.K. Cheung, E. Roca. Economic Modelling 29 (3), 684-690 (2012). DOI: 10.1016/j.econmod.2012.01.015.

13. P.V. Ngo. Journal of Macroeconomics 44, 191-207 (2015). DOI: 10.1016/j.jmacro. 2015.02.005.

14. H.C. Bjomland, D.H. Jacobsen. Journal of Financial Stability 6 (4), 218-229 (2010). DOI: $10.1016 /$ j.jfs.2010.02.001.

15. P. Karpestam, S. Johansson. Journal of Housing Economics 46, 101627 (2019). DOI: 10.1016/j.jhe.2019.03.004.

16. G.M. Zagidullina, A.I. Romanova, M.R. Akhmerov, A.A. Murafa. Izvestija KGASU, 4 (26), 296-302 (2013).

17. T. Engsted, T.Q. Pedersen. Journal of International Money and Finance 53, 257-275 (2015). DOI: 10.1016/j.jimonfin.2015.02.001.

18. A. Gazzani. Review of Economic Dynamics 36, $46-72$ (2020). DOI: 10.1016/j.red.2019.08.001.

19. L. Tupenaite, L. Kanapeckiene, J. Naimaviciene. Procedia Engineering 172, 1169-1175 (2017). DOI: 10.1016/j.proeng.2017.02.136.

20. L. Gaspareniene, R. Remeikiene, A. Skuka. Intellectual Economics 10 (2), 122-127 (2016). DOI: 10.1016/j.intele.2017.03.005.

21. L. Liu, L. Wu. Socio-Economic Planning Sciences 72, 100916 (2020). DOI: 10.1016/j.seps.2020.100916.

22. H. Alqaralleh, A. Canepa. Economic Modelling 92, 257-267 (2020). DOI: 10.1016/j.econmod.2020.01.005. 
23. A. Canepa, E. Zanetti Chini. Journal of Empirical Finance 37, 91-103 (2016). DOI: 10.1016/j.jempfin.2016.02.011.

24. I.E. Fayzullin. Electronic economic newsletter of the Republic of Tatarstan 1, 249-263 (2012).

25. The Institute for Urban Economics: http://www.urbaneconomics.ru/research/analytics/ dinamika-dostupnosti-zhilya-v-rossii-v-1998-2019-gg.

26. Statistical Review of the Republic of Tatarstan: http://tatstat.gks.ru/.

27. The Central Bank of the Russian Federation: http://www.cbr.ru/.

28. Statistical Review of the Russian Federation: http://www.gks.ru/. 